\title{
Ternary and Higher Order Iron Phase Diagram Updates
}

Ternary and quaternary iron phase diagrams have been critically evaluated and published as a series of monographs by the cooperating societies: ASM International, Indian Institute of Metals, and Institute of Materials (United Kingdom). Updates on multicomponent iron phase diagrams are published regularly in this journal.

This issue carries updates on eight ternary systems: Al-Fe-U, Al-Fe-Zr, Fe-Hf-Zr, Fe-Nb-Zr, Fe-Pu-U, Fe-Si-U, Fe-Sn-U, and Fe-U-Zr, and the quaternary system Fe-Pu-U-Zr. Plutonium, uranium, and/or zirconium form alloy fuels that are used in fast nuclear reactors. The fuel, as it swells, can come into contact with the cladding material (usually stainless steel). The ensuing metallurgical interaction can degrade the cladding. This problem is of major concern to the nuclear industry.

\author{
V. Raghavan \\ Editor \\ Phase Diagrams of \\ Ternary Iron Alloys \\ Parts 1, 2, 3, 5, and 6
}

